\title{
शिमला जनपद के अवनद्ध व सुषिर लोक वाद्य
}

\section{Pawan Kumar}

Research Scholar, Department of Music, Himachal Pradesh University, Shimla

सारांश- शिमला जनपद हिमाचल प्रदेश का एक बहुत ही खुबसूरत ज़िला है जो अपनी लोक संस्कृति व प्राकृतिक सुन्दरता के लिए विश्वविख्यात है और साथ ही यहां के लोक संगीत की भी अपनी विशिष्ट पहचान है। यहां के लोक संगीत के अर्न्तगत लोकगीत गायन, लोकवाद्य वादन व लोकनृत्य तीनों समान रूप से सम्मिलित है। प्रस्तुत शोध पत्र में लोकवाद्य के अर्त्तगत जनपद के अवनद्ध व सुषिर वाद्य यंत्रों पर दृष्टिपात किया गया है। जनपद के अवनद्ध व सुषिर वाद्य के अर्न्तगत आने वाल वाद्यों यंत्रों का परिचय, बनावट विधि व वादन प्रक्रिया के ऊपर प्रकाश डालने की कोशिश की गई है।

\section{भूमिका}

शिमला जनपद का अधिकांश भाग आर्य सभ्यता से प्रभावित रहा है। महाभारत काल के पाण्डवों से सम्बन्धित तो यहां अनेक प्रमाण भी मिलते हैं। अतः यहां के लोगवाद्यों की उत्पति भी भारतवर्ष के अन्य भागों के सदृश हुई होगी, ऐसा आभास मिलता है। भारतीय प्रचलित संगीत वाद्यों को तत्, अवनद्ध, सुषिर और घनवाद्य चार भागों में वर्गीकृत किया गया है। शिमला जनपद में प्रचलित सभी वाद्य इसी वर्गीकरण के अर्त्तगत आ जाते हैं। शिमला अंचल में तार वाले वाद्य न के बराबर है। अतः यहां के वाद्यों को अवनद्ध, सुषिर तथा घनवाद्य, तीन प्रकार के वर्गों में रख सकते हैं। लेकिन शिमला जनपद में प्रचलित 'लोकरामायण' की पंक्तियों में कुछ वाद्यों का वर्णन आता है जिसमें "किन्द्री" शब्द भी आया है जोकि सम्भवतः "किन्नरी वीणा" के लिए प्रयुक्त हुआ है परन्तु वर्तमान में ऐसे किसी वाद्य के इस जनपद में दर्शन नहीं होते, पहाड़ी लोक रामायण की कुछ पंक्तियां देखिए :

रामो री आरती हुई रे

जोड़िए नौबजा बाजी रे

ढौप(ढप) लागौ मुरली रा बाजा रे

खारी कौरा दारूए मुहाला रे

लाइयौ सुनुए किन्द्री, रूणो मोरो ले हे गुणी

कुणियो रामो रे भीतरी मेरी किन्द्री शुणो 
ऐसा लगता है कि प्राचीन समय में यहां तत् वाद्यों के कुछ प्रकार रहे होंगे। जैसे- किन्दरी, किंगरी, किन्नरी। इसका प्रयोग भी केवल जोगी साधु इत्यादि ही धार्मिक उत्सवों पर अथवा नृत्य के समय करते होंगे परन्तु वर्तमान समय में इन सब का प्रयोग सुनने को नहीं मिलता।

\section{शिमला जनपद के अवनद्ध वाद्य}

अवनद्ध वाद्य वे वाद्य होते हैं जो जानवरों की खाल से मढ़े या बनाए जाते हैं। किसी धातु या लकड़ी के खोखले भाग को पशुओं की खाल से मढ़ा जाता है जिससे वे वादन के योग्य बनते हैं। ये वाद्य अधिकतर लय व ताल के लिए प्रयोग में लाए जाते हैं। अवनद्ध वाद्यों का प्रयोग "मिथकों" में शिवजी के डमरू से जोड़ा जाता है। कहते हैं कि शिव ने अपने विराट नृत्य के समय डमरू का प्रयोग किया था। शिमला जनपद में इस श्रेणी के अर्त्तगत प्रयोग किए जाने वाले वाद्य इस प्रकार है:
1. डमरू (गुज्जु)
2. खंजरी (डफ)
3. नगाड़ा
4. ढोल(ढोलकी)

\section{डमरू (गुज्जु)}

इसका बीच का सिरा पतला तथा किनारों के दोनों सिरे बराबर गोल आकार के होते हैं। इसके दोनों सिरों पर चर्मपत्र कसे रहते हैं तथा इसकी कमर में धातु, लकड़ी अथवा डाट का एक छोटा सा गोला रस्सी से बांधा जाता है जोकि हिलाने पर दोनों

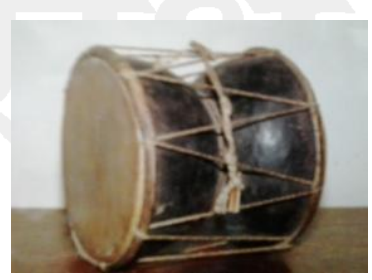
ओर बंधे चर्म पत्र से टकरा जाता है तथा इसे गायन, वादन तथा नृत्य तीनों के लिए ही प्रयोग जाता है। दाए अथवा बांए घुमाने से इससे इच्छित ताल निकाली जाती है। इसे गुज्जु, डांरू आदि नाम से भी पुकारा जाता है। इसकी लम्बाई $6^{\prime \prime}$ से एक फुट होती है।

\section{खंजरी (डफ)}

खंजरी या डफ ये दोनों मिलते जुलते वाद्य हैं। "डफ" आकार में कुछ बड़ा होता है अर्थात इसका घेरा लगभग तीन फुट तथा चौड़ाई 4 से 6 इंच तक होती है। इसे हाथ के अतिरिक्त लकड़ी के छोटे-छोटे डण्डों से भी बजाने की प्रथा है।

खंजरी का आकार लगभग $10^{\prime \prime}$ से $12^{\prime \prime} 14^{\prime \prime}$ इंच तक तथा चौड़ाई लगभग 2 " से $3^{\prime \prime}$ इंच तक होती है। इसके बाहरी भाग पर $1 / 2^{\prime \prime}$ अथवा $1^{\prime \prime} 2^{\prime \prime}$ इंच तक का मोटा चौखट होता है जिसमें लगभग समान दूरी पर "टीन" के कुछ गोलाकार टुकड़े तार से जोड़े जाते हैं। 'खंजरी' को(दाए या 


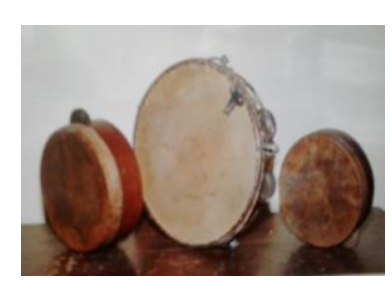
बांए) हाथ की उँगली और हथेली के परस्पर सहयोग से थपक लगाकर बजाया जाता है जिससे ये 'टीन' के टुकड़े 'छनछनाहट' पैदा करते हैं। लोकसंगीत की इच्छित लय के साथ इसे मिलाया जाता है।

धार्मिक तथा सभी प्रकार के अन्य लोकगीतों एवं नृत्यों के साथ इसका प्रयोग देखने को मिलता है। वैसे अपनी सरल बनावट तथा हल्के छोटे आकार के कारण भी वर्तमान में इसका बहुत प्रयोग होता है। यह सम्भवतः पुराने समाघात लोकवाद्यों में से एक है।

\section{नगाड़ (नगारा)}

नगाड़ा' प्राचीन भेरी और दुन्दुभी का ही रूप लगता है तथा इसे प्राचीन लोकवाद्यों का ही एक प्रकार माना गया है। इसके विभिन्न आकार होते हैं। अर्द्ध गोलाकार रूप के नगाड़े ताँबे, पीतल, लोहे अथवा चाँदी के बने होते हैं। इन पर तबले की तरह ही एक मजबूत 'खाल' (बैल, बकरे या हिरण की) मजबूत रस्सियों से अथवा चमड़े की डोरी से कसी जाती है। लकड़ी के बने छोटे डण्डों से उन्हें बजाया जाता है और बड़ी गहरी, मधुर सी ध्वनि इससे निकलती है। 'ऊपरी महासू' क्षेत्रों में कई स्थानों पर नगारों के 'जोड़े' भी बनाए जाते हैं तथा दोनों से ही अलग-अलग घ्वनि निकलती है। देवयात्रा के समय एक आदमी इन्हें पीठ पर उठाता है तथा वादक इन्हें परम्परागत ढंग की ताल में विभिन्न लयकारियों में कुश्लता से बजाता है। कई मन्दिरों में बड़े-बड़े नगारे भी रखे जाते हैं। 'पूजा' इत्यादि के अवसर पर इन्हें बजाया जाता है। लोकनृत्य, लोकगीत शादी-ब्याह धार्मिक कृत्य इत्यादि के अवसर पर बाकी अन्य वाद्यों के साथ नगारों का वादन दिव्य अनुभूति प्रदान करता है। मन्दिरों में प्रायः सभी वाद्य बजाए जाते हैं। महाभारत में एक कथा है - एक बार जयद्रथ ने वृषभ रूपधारी एक राक्षस को मारकर उसकी चमड़ी मढ़कर तीन 'नगारे' बनवाए और उनको एक बार बजाने से एक माह तक 'नाद' गुँजता था।

\section{ढोल और ढोलकी}

इन दोनों में केवल आकार का अन्तर है। ढोल का ही छोटा रूप ढोलकी है। ढोलकी छोटी आकार के कारण कीर्तन इत्यादि में भी बजाई जा सकती है तथा इधर-उधर ले जाने में भी समस्या नही होती। इसके छोटे आकार के कारण इस पर होथों से ही विभिन्न लयकारियां, तालों के प्रकार बजाए जा सकते हैं परन्तु 'ढोल' का आकार बड़ा होने के कारण उस पर हाथों से सीधे ही 
लयकारियां व तालों के प्रकार बजाने सुगम नहीं है। नगारे के साथ 'ढोल' का होना आवश्यक है। लोकनृत्य तथा लोकसंगीत के अवसर पर ढोल पर पड़ने वाली थाप विशेष आनन्दानुभूति करवाने में सक्षम है। ढोल प्राय: आम, आक, सानन की लकड़ी तथा पीतल अथवा अन्य धातु के होते हैं। गोलाकार सी विशेष प्रकार की लकड़ी में खोल बनाकर, चर्मपत्र, इसके दोनों किनारों पर सुत की पक्की रस्सी या चमड़े की पट्टी से कसकर बांधे जाते हैं। बांई ओर की खाल पर धूप, कांसे का बूर लोहे का बूर आदि लेकर बकरी के दूध में पीसकर तैयार किया जाता है तथा खाल पर विशेष ढंग से लगाया जाता है। इस प्रकार इसकी गूँज में एक गहरापन, भारीपन सा आ जाता है। इसे

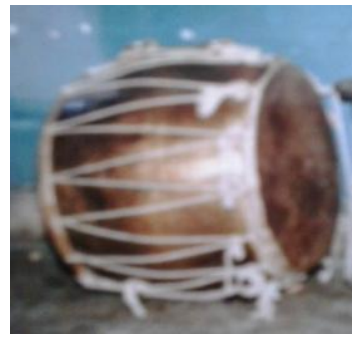
अधिकतर बांई ओर से हॉकी की तरह मुड़ी हुई छोटी लकड़ी से बजाया जाता है तथा दांई ओर हाथ द्वारा प्रहार किया जाता है। प्राय: 'ढोल' को गले में लटकाने के लिए पक्के 'सूत' की रस्सी का प्रयोग किया जाता है। नगारे के साथ का यह पूरक वाद्य है तथा इसे पूर्णता प्रदान करता है।

\section{शिमला जनपद के सुषिर वाद्य}

इसके अर्न्तगत 'फूंक' से बजाए जाने वाल वाद्य आते हैं। शिमला जनपद में प्रचलित सुषिर वाद्य इस प्रकार हैं :- 1. नरसिंगा 2. करनाल 3. बांसुरी 4. शहनाई 5. शंख

\section{नरसिंगा}

यह अंग्रेजी भाषा के अक्षर एस(S) आकार का होता है तथा तांबे की बनी दो भागों वाली नाली से निर्मित किया जाता है। फूंक देने वाली पतली जगह पर इसमें एक छोटा सा सुराख होता है और इसका आकार 8 " से $10^{\prime \prime}$ इंच तक खुला होता है। लोकनृत्य तथा लोकगीत गाते समय, दोनों में ही इसे कुछ-कुछ अन्तराल पर बजाया जाता है। विवाह के अवसर पर तथा पूजा अर्चना के अवसर पर इसका प्रयोग किया जाता है।

\section{करनाल}

'कर' और 'नाल' अर्थात हाथ में ली जाने वाली नलिका। शिमला जनपद का यह भी एक प्रमुख वाद्य है। इसका सिरा तंग तथा एक खुला होता है। जैसे कोई लम्बी सी कीप होती है। इसको भी दो नलियों से जोड़ा जाता है। यह अधिकतर पीतल अथवा चाँदी का होता है तथा फूंक द्वारा इसे गायन-वादन अथवा नृत्य क्रियाओं के मध्य कुछ-कुछ अन्तराल

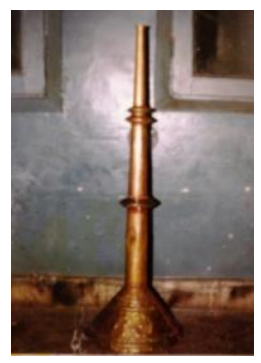


से बजाया जाता है। इसकी ध्वनि में एक विशेष संवाद रहता है। अधिकतर "सा-प" अथवा "सा-सां" स्वरों का ही ध्वनिकरण इसमें सुनाई पड़ता है।

\section{बांसुरी}

स्थानीय बोली में इसे "बिशड़ी" भी कहतें हैं। भारतीय संगीत में बांसुरी का सम्बन्ध मुरली मनोहर श्री कृष्ण भगवान से जोड़ा जाता है। यह प्राचीन लोकवाद्य है। 'बांस' या लोहे

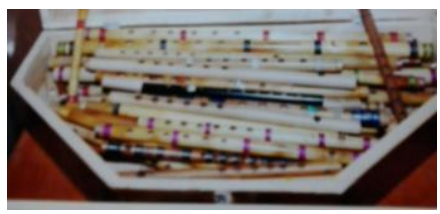
की नलिका द्वारा निर्मित बांसुरी में 6 से 8 तक सीधी पंक्ति में सुराख होते है। एक ओर से सुराख बन्द रहता है। कुछ बांसुरियां सीधी तरह से तथा कुछ बांसुरियां किनारे से बजाई जाती है। फूंक देने के लिए निर्मित सुराख सीधी पंक्ति के अन्य सुराखों से कुछ दूर ऊपर की ओर स्थित रहता है। इसके लिए नियंत्रित श्वास क्रिया तथा उंगलियों के संचालन का अच्छा अभ्यास होना आवश्यक है। शिमला अंचल में प्रचलित अधिकांश लोकगीतों में बांसुरी का सुन्दर प्रयोग हुआ है।

\section{शहनाई}

स्थानीय बोली में इसे 'सरनाई' भी कहतें हैं तथा शिमला जनपद में इसे बांसुरी से कुछ अधिक महत्ता प्राप्त है। सम्भवतः इसकी बुलन्द, ऊँची आवाज ही इसकी प्रसिद्धि का कारण रहा होगा। इसे "तुरी" जिन्हें हेसी, बजन्तरी भी कहते हैं तथा जो अधिकतर अनुसूचित जाति से सम्बन्ध रखते हैं। इसका विवाह, धार्मिक पूजा अर्चना तथा मेलों त्य ोहारों के अवसरों पर बहुधा प्रयोग करते हैं नृत्य के लिए तो शहनाई का अलग ही महत्त्व है। यह नाली की तरह साफ की हुई लकड़ी अथवा कहीं-कहीं धातु की बनी होती है और कुछ चौड़े सिरे की गोलाकार घण्टी सी लगी होती है। यह एक से दो फूट तक लम्बी होती है जिसमें 8 या 9 सुराख होते है। ऊपर के 7 सुराख बराबर बजाए जाते हैं तथा शेष सुराख वादक की इच्छा एवं सुविधा पर निर्भर है कि उन्हें स्वराघात के लिए खुले रखें अथवा बंद रखें। शहनाई वादन जटिल प्रक्रिया है और इसके लिए अनवरत उचित अभ्यास की आवश्यकता रहती

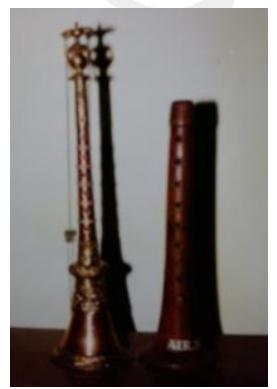
है। इसमें प्रयोग की जाने वाली कम्पिका पालाघास की बनी होती है। उसे बजाने वाले सिरे पर जोड़ते हैं। एक अन्य 'कम्पिका'(पत्ती) और हाथी दाँत की सुई की तरह एक छोटी सी डण्डिका को इस कम्पिका के साथ जोड़ा जाता है ताकि इसका सामंजस्य बना रहे। शहनाई वादक लोकनृत्य के समय विशेष आकर्षण का केन्द्र बने रहते हैं। 


\section{शंख}

घरों तथा पूजास्थलों पर बहुधा मंगलसूचक ध्वनि के लिए इसका प्रयोग होता है। शिमला ज़िला के अधिकतर स्थानों पर मृत्यु के समय भी "उल्टा शंख" (एक सीधी सी ध्वनि) बजाने का प्रचलन है।

\section{निष्कर्ष}

इस प्रकार हम निष्कर्षतः कह सकते हैं कि शिमला जनपद के अवनद्ध व सुषिर वाद्यों का अपना एक पारम्परिक और विशेष गौरसमयी स्वरूप है। ये वाद्य धनिक एवं अभिजात्य वर्ग के लोगों की सम्पति नहीं, अपितू साधारण, अनपढ़ पहाड़ी वर्ग के लोगों की अमूल्य सम्पदा है जो इनकी शास्त्रीय मान्यताओं एवं मापदण्डों से अनभिज्ञ हैं। प्राचीन काल से ही लोकवाद्य शास्त्रीय संगीत के प्रेरक रहे हैं। तार्किक दृष्टि से भी देखें तो हमें मालूम होगा कि शास्त्रीय संगीत में प्रयुक्त वाद्यों का उद्भव और विकास लोकवाद्यों के आधार पर ही हुआ है।

लोकसंगीत और लोकवाद्य शास्त्रीय संगीत से प्रभावित नहीं हुए अपितू शास्त्रीय संगीत के लिए प्रत्यक्ष एवं परोक्ष रूप से इनसे प्रेरणा मिलती रही। आधुनिक प्रचलित सांगीतिक रूपों तथा शास्त्रीय संगीत की शैलीयों में कई बार लोकवाद्यों की अवहेलना हुई है परन्तु परम्परागत रूप में अपने अस्तित्व पर गौरव करके यहां के लोकवाद्यों ने सदैव जनजीवन को एकसुत्र में बांधने का सार्थक प्रयास किया और शास्त्रीय संगीत के व्यापक प्रचार-प्रसार एवं लोकप्रियता के बावजुद भी लोकवाद्यों का एक सम्माननीय अस्तित्व है जिनका अपना परम्परागत पूजनीय रूप है।

\section{सन्दर्भ-ग्रन्थ सूची}

गर्ग, नन्दलाल, हिमाचल के प्राचीनतम वाद्य, संगीत नाटक अकादमी कनिष्क पब्लिशर्स नई दिल्ली,2009

मिश्र, लालमणि, भारतीय संगीत वाद्य, भारतीय ज्ञान पीठ प्रकाशन उत्तर प्रदेश,1973 शर्मा, डॉ० महेन्द्र प्रसाद, अवनद्ध वाद्य(सिद्धान्त एवं वादन परम्परा), अभिषेक पब्लिकेशन चण्डीगढ़,2002 मिश्रा, डॉ० अरूणा, भारतीय कण्ठ संगीत और वाद्य संगीत, कनिष्का पब्लिशर्स नई दिल्ली,2002 